\title{
Essential self-adjointness for combinatorial Schrödinger operators II- Metrically non complete graphs
}

\author{
Yves Colin de Verdière* \\ Nabila Torki-Hamza ${ }^{\dagger}$ \\ Françoise Truc ${ }^{\ddagger}$
}

March 3, 2013

\begin{abstract}
We consider weighted graphs, we equip them with a metric structure given by a weighted distance, and we discuss essential self-adjointness for weighted graph Laplacians and Schrödinger operators in the metrically non complete case.
\end{abstract}

\section{Introduction}

This paper is a continuation of [To] which contains some statements about essential self-adjointness of Schrödinger operators on graphs. In [To], it was proved that on any metrically complete weighted graph with bounded degree, the Laplacian is essentially self-adjoint and the same holds for the Schrödinger operator provided the associated quadratic form is bounded from below. These results remind those in the context of Riemannian manifold in [Ol] and also in [B-M-S],

\footnotetext{
${ }^{0}$ Keywords: metrically non complete graph, weighted graph Laplacian, Schrödinger operator, essential selfadjointness.

${ }^{0}$ Math Subject Classification (2000): 05C63, 05C50, 05C12, 35J10, 47B25.

${ }^{*}$ Grenoble University, Institut Fourier, Unité mixte de recherche CNRS-UJF 5582, BP 74, 38402-Saint Martin d'Hères Cedex (France); yves . colin-de-verdiere@ujf-grenoble.fr; http://www-fourier.ujf-grenoble.fr//ycolver/

'Unité de recherches Mathématiques et Applications, 05/UR/15-02, Faculté des Sciences de Bizerte de l'Université de Carthage, 7021-Bizerte; ISIG-K, Université de Kairouan, 3100Kairouan; Tunisie; nabila.torki-hamza@fsb.rnu.tn; natorki@yahoo.fr

${ }^{\ddagger}$ Grenoble University, Institut Fourier, Unité mixte de recherche CNRS-UJF 5582, BP 74, 38402-Saint Martin d'Hères Cedex (France); francoise.truc@ujf-grenoble.fr; http://www-fourier.ujf-grenoble.fr/ trucfr/
} 
[Shu1, Shu2]. There are many recent independent researches in locally finite graphs investigating essential self-adjointness (see [Jor, Go-Sch], [Ma]), and relations between stochastic completeness and essential self-adjointness ( see [We], Woj2 as well as the thesis [Woj1]). Similar results have been extended for arbitrary regular Dirichlet forms on discrete sets in [Ke-Le-2] which is mostly a survey of the original article [Ke-Le-1]. More recently the paper [ $\mathrm{Hu}$ is devoted to the stability of stochastic incompleteness, in almost the same setup as in Ke-Le-1. Here, we will investigate essential self-adjointness mainly on metrically non complete locally finite graphs.

Let us recall that a weighted graph $G$ is a generalization of an electrical network where the set of vertices and the set of edges are respectively weighted with positive functions $\omega$ and $c$. For any given positive function $p$, a weighted distance $d_{p}$ can be defined on $G$. Thus we have the usual notion of completeness for $G$ as a metric space.

The main result of Section 3 states that the weighted graph Laplacian $\Delta_{\omega, c}$ (see the definition (1) below) is not essentially self-adjoint if the graph is of finite volume and metrically non complete (here the metric $d_{p}$ is defined using the weights $\left.p_{x, y}=c_{x, y}^{-\frac{1}{2}}\right)$. The proof is derived from the existence of the solution for a Dirichlet problem at infinity, established in Section 2.

In Section 4, we establish some conditions implying essential self-adjointness. More precisely, defining the metric $d_{p}$ with respect to the weights $p_{x, y}$ given by $p_{x, y}=\left(\min \left\{\omega_{x}, \omega_{y}\right\}\right) c_{x, y}^{-\frac{1}{2}}$, and addressing the case of metrically non complete graphs, we get the essential self-adjointness of $\Delta_{\omega, c}+W$ under the assumptions that the potential $W$ is bounded from below by $N / 2 D^{2}$, where $N$ is the maximal degree and $D$ the distance to the boundary, and that the graph has a regularity property. We use for this result a technical tool deduced from Agmon-type estimates and inspired by the nice paper [Nen], see also [Col-Tr].

We discuss in Section 5 the case of star-like graphs. Under some assumptions on $a$, we prove that for any potential $W, \Delta_{1, a}+W$ is essentially self-adjoint using an extension of Weyl's theory to the discrete case. In the particular case of the graph $\mathbb{N}$, the same result had been proved in $[\mathrm{Ber}$ (p.504) in the context of Jacobi matrices. We give some examples in Subsection 5.3 to illustrate the links between the previous results. Moreover we establish the sharpness of the conditions of Theorem 4.2.

The last Section is devoted to Appendix A dealing with Weyl's limit point-limit circle criteria (see [RS]) in the discrete case as well as in the continuous case, and to Appendix B including the unitary equivalence between Laplacians and Schrödinger operators [To] used repeatedly in Subsection 5.3 .

Let us start with some definitions.

$G=(V, E)$ will denote an infinite graph, with $V=V(G)$ the set of vertices and $E=E(G)$ the set of edges. We write $x \sim y$ for $\{x, y\} \in E$.

The graph $G$ is always assumed to be locally finite, that is any $x \in V$ has a finite 
number of neighbors, which we call the degree (or valency) of $x$. If the degree is bounded independently of $x$ in $V$, we say that the graph $G$ is of bounded degree. The space of real functions on the graph $G$ is denoted

$$
C(V)=\{f: V \longrightarrow \mathbb{R}\}
$$

and $C_{0}(V)$ is the subspace of functions with finite support.

We consider, for any weight $\omega: V \longrightarrow] 0,+\infty[$, the space

$$
l_{\omega}^{2}(V)=\left\{f \in C(V) ; \sum_{x \in V} \omega_{x}^{2} f^{2}(x)<\infty\right\} .
$$

It is a Hilbert space when equipped with the inner product:

$$
\langle f, g\rangle_{l_{\omega}^{2}}=\sum_{x \in V} \omega_{x}^{2} f(x) \cdot g(x)
$$

For any $\omega: V \longrightarrow] 0,+\infty[$, and $c: E \longrightarrow] 0,+\infty[$, the weighted graph Laplacian $\Delta_{\omega, c}$ on the graph $G$ weighted by the conductance $c$ on the edges and by the weigth $\omega$ on the vertices, is defined by:

$$
\left(\Delta_{\omega, c} f\right)(x)=\frac{1}{\omega_{x}^{2}} \sum_{y \sim x} c_{x, y}(f(x)-f(y))
$$

for any $f \in C(V)$ and any $x \in V$. If $\omega \equiv 1$, we have

$$
\left(\Delta_{1, a} f\right)(x)=\sum_{y \sim x} a_{x, y}(f(x)-f(y)) .
$$

Definition 1.1 Let $p: E \longrightarrow] 0,+\infty\left[\right.$ be given, the weighted distance $d_{p}(\leq+\infty)$ on the weighted graph $G$ is defined by

$$
d_{p}(x, y)=\inf _{\gamma \in \Gamma_{x, y}} L(\gamma)
$$

where $\Gamma_{x, y}$ is the set of the paths $\gamma: x_{1}=x, x_{2}, \cdots, x_{n}=y$ from $x$ to $y$. The length $L(\gamma)$ is computed as the sum of the p-weights for the edges of the path $\gamma$ :

$$
L(\gamma)=\sum_{1 \leq i \leq n} p_{x_{i}, x_{i+1}}
$$

In particular, if $x$ and $y$ are in distinct connected components of $G, d_{p}(x, y)=\infty$. We say that the metric space $\left(G, d_{p}\right)$ is complete when every Cauchy sequence of vertices has a limit in $V$.

Definition 1.2 We denote by $\hat{V}$ the metric completion of $\left(G, d_{p}\right)$ and by $V_{\infty}=$ $\widehat{V} \backslash V$ the metric boundary of $V$.

Definition 1.3 If $G$ is a non finite graph and $G_{0}$ a finite sub-graph of $G$, the ends of $G$ relatively to $G_{0}$ are the non finite connected components of $G \backslash G_{0}$. 


\section{The Dirichlet problem at infinity}

We will use in this section the distance $d_{p}$ defined using the weights $p_{x, y}=c_{x, y}^{-\frac{1}{2}}$. Let us consider the quadratic form

$$
Q(f)=\sum_{\{x, y\} \in E} c_{x, y}(f(x)-f(y))^{2}+\sum_{x \in V} \omega_{x}^{2} f(x)^{2}
$$

which is formally associated to the operator $\Delta_{\omega, c}+\operatorname{Id}$ on $l_{\omega}^{2}$. We will need the following result which is close to lemma 2.5 in [Jo-Pe-2]:

Lemma 2.1 For any $f: V \rightarrow \mathbb{R}$ so that $Q(f)<\infty$ and for any $a, b \in V$, we have

$$
|f(a)-f(b)| \leq \sqrt{Q(f)} d_{p}(a, b) .
$$

Proof.-

For any $\{x, y\} \in E,|f(x)-f(y)| \leq \sqrt{Q(f)} / \sqrt{c_{x, y}}$. For any path $\gamma$ from $a$ to $b$, defined by the vertices $x_{1}=a, x_{2}, \cdots, x_{n}=b$, we have $|f(a)-f(b)| \leq \sqrt{Q(f)} L(\gamma)$. Taking the infimum of the righthandside with respect to $\gamma$ we get the result.

Remark 2.1 Lemma 2.1 implies that any function $f$ with $Q(f)<\infty$ extends to $\hat{V}$ as a Lipschitz function $\hat{f}$. We will denote by $f_{\infty}$ the restriction of $\hat{f}$ to $V_{\infty}$.

Theorem 2.1 Let us assume that $\left(V, d_{p}\right)$ is non complete. Let $f: V \rightarrow \mathbb{R}$ with $Q(f)<\infty$, then there exists a continuous function $F: \hat{V} \rightarrow \mathbb{R}$ which satisfies both conditions:

(i) $(F-f)_{\infty}=0$

(ii) $\left(\Delta_{\omega, c}+1\right)\left(F_{\mid V}\right)=0$.

Moreover, such an $F$ satisfies $Q(F)<\infty$ and $F \in l_{\omega}^{2}$.

If $\hat{V}$ is compact, such an $F$ is unique.

Proof.-

We will denote by $A_{f}$ the affine space of continuous functions $G$ : $\hat{V} \rightarrow \mathbb{R}$ which satisfy $Q(G)<\infty$ and $(G-f)_{\infty}=0$.

$Q$ is lower semi-continuous for the pointwise convergence on $V$ as defined by $Q=\sup Q_{\alpha}$ with $Q_{\alpha}(f)=$ sum of a finite number of terms in $Q$.

Let $Q_{0}:=\inf _{G \in A_{f}} Q(G)$ and $G_{n}$ be a corresponding minimizing sequence. The $G_{n}$ 's are equicontinuous and pointwise bounded. From 
Ascoli's Theorem, this implies the existence of a locally uniformly convergent subsequence $G_{n_{k}} \rightarrow F$. Using semi-continuity, we have $Q(F)=Q_{0}$.

If $x \in V$ and $\delta_{x}$ is the Dirac function at the vertex $x$, we have

$$
\left.\frac{d}{d t}\right|_{t=0} Q\left(F+t \delta_{x}\right)=2 \omega_{x}^{2}\left[\left(\Delta_{\omega, c}+1\right) F(x)\right]
$$

and this is equal to 0 , because $F$ is a minimum of $Q$ restricted to $A_{f}$.

Uniqueness is proved using a maximum principle: let us assume that there exists a non zero continuous $F$ with $F_{\infty}=0$, then, changing, if necessary, $F$ into $-F$, there exists $x_{0} \in V$ with $F\left(x_{0}\right)=$ $\max _{x \in V} F(x)>0$. The identity (ii) evaluated at the vertex $x_{0}$ gives a contradiction.

\section{Not essentially self-adjoint Laplacians}

Theorem 3.1 Let $\Delta_{\omega, c}$ be a weighted graph Laplacian and assume the following conditions:

(i) $\left(G, d_{p}\right)$ with $p_{x, y}=c_{x, y}^{-\frac{1}{2}}$ is NON complete,

(ii) there exists a function $f: V \rightarrow \mathbb{R}$ with $Q(f)<\infty$ and $f_{\infty} \neq 0$.

Then $\Delta_{\omega, c}$ is not essentially self-adjoint.

Proof.-

Because $\Delta_{\omega, c}$ is $\geq 0$ on $C_{0}(V)$, it is enough (see Theorem X.26 [RS]) to build a non zero function $F: V \rightarrow \mathbb{R}$ which is in $l_{\omega}^{2}(V)$ and satisfies

$$
\left(\Delta_{\omega, c}+1\right) F=0 \text {. }
$$

The function $F$ given by Theorem 2.1 will be the solution of equation (2) the limit of which at infinity is $f_{\infty}$.

Remark 3.1 The assumptions of Theorem 3.1 are satisfied if $\left(G, d_{p}\right)$ is non complete and $\sum \omega_{y}^{2}<\infty$ : it is enough to take $f \equiv 1$.

They are already satisfied if $G$ has a non complete "end" of finite volume.

Remark 3.2 Theorem 3.1 is not valid for the Riemannian Laplacian: if $X$ is a closed Riemannian manifold of dimension $\geq 4, x_{0} \in X$ and $Y=X \backslash x_{0}$, the Laplace operator on $Y$ is essentially self-adjoint (see [Col1]) and $Y$ has finite volume.

Question 3.1 In Theorem 3.1, what is the deficiency index of $\Delta_{\omega, c}$ in terms of the geometry of the weighted graph? 


\section{Schrödinger operators for metrically non com- plete graphs}

We now discuss essential selfadjointness for Schrödinger operators of the type $H=\Delta_{\omega, c}+W$ on a graph $G$ in the following setup: we define $\alpha_{x, y}=\min \left\{\omega_{x}, \omega_{y}\right\}$

and we assume that $\left(G, d_{p}\right)$, with $p_{x, y}=\frac{\alpha_{x, y}}{\sqrt{c_{x, y}}}$, is non complete as a metric space. It means that there exist Cauchy sequences of vertices without limit in the set $V$. We will assume that $G$ is of bounded degree, and we denote the upper bound by $N$. We will need also to assume a regularity property for $\left(G, d_{p}\right)$.

\subsection{Regularity property for metric graphs}

Definition 4.1 For a vertex $x \in V$, we denote by $D(x)$ the distance to the boundary $V_{\infty}$ defined by

$$
D(x)=\inf _{z \in V_{\infty}} d_{p}(x, z) .
$$

Lemma 4.1 We have, for any edge $\{x, y\}$,

$$
|D(x)-D(y)| \leq d_{p}(x, y) \leq \frac{\min \left\{\omega_{x}, \omega_{y}\right\}}{\sqrt{c_{x, y}}} .
$$

Definition 4.2 If $A$ is a subset of $V$, the boundary $\partial A$ of $A$ is the set of the vertices $x \in A$ so that there exists $y \in V \backslash A$ with $\{x, y\} \in E$.

Definition 4.3 Let $\varepsilon>0$ be given and $X_{\varepsilon}$ be defined by

$$
X_{\varepsilon}=\{x \in V \mid D(x) \geq \varepsilon\} .
$$

We say that the graph $\left(G, d_{p}\right)$ is regular if, for any sufficiently small $\varepsilon$, any bounded subset of $\partial X_{\varepsilon}$ (for the metric $d_{p}$ ) is finite.

The main property of the regular graphs that we will use is:

Proposition 4.1 If $\left(G, d_{p}\right)$ is regular, then closed and bounded subsets of $X_{\varepsilon}$ are finite.

Proof.-

Let $A$ be a closed and bounded subset of $X_{\varepsilon}$.

Let $\Gamma=\left(V^{\prime}, E^{\prime}\right)$ be the graph with vertices $V^{\prime}=A$ and edges $E^{\prime}=E_{1}^{\prime} \cup E_{2}^{\prime}$ defined as follows:

(i) edge $\{x, y\}$ is in $E_{1}^{\prime}$ iff $\{x, y\} \in E$ and $x, y \in A$,

(ii) edge $\{x, y\}$ is in $E_{2}^{\prime}$ iff $\{x, y\} \notin E$ and $x, y \in A \cap \partial X_{\varepsilon}$. 
Note that the edges $E_{1}^{\prime}$ already exist in $G=(V, E)$, while the set $E_{2}^{\prime}$ represents new edges.

The graph $\Gamma$ is equipped with the following weight:

$$
p_{x, y}^{\prime}=\left\{\begin{array}{cl}
p_{x, y}, & \{x, y\} \in E_{1}^{\prime}, \\
d_{p}(x, y), & \{x, y\} \in E_{2}^{\prime} .
\end{array}\right.
$$

Clearly, the distances $d_{p^{\prime}}$ and $d_{p}$ coincide on $A$. If $\left(x_{n}\right)_{n \in \mathbb{N}}$ is a Cauchy sequence for $\left(A, d_{p}\right)$, it has a limit in $\hat{V}$ which lies in $A$ because $A$ is closed in $\hat{V}$. Hence, the graph $\left(\Gamma, d_{p^{\prime}}\right)$ is complete. Since $A \cap \partial X_{\varepsilon}$ is a bounded subset of $\partial X_{\varepsilon}$, by regularity property it follows that $A \cap \partial X_{\varepsilon}$ is finite; hence, the set $E_{2}^{\prime}$ is finite. This, together with local finiteness of $G=(V, E)$, shows that $\Gamma=\left(V^{\prime}, E^{\prime}\right)$ is locally finite. Since $A$ is closed and bounded with respect to the metric $d_{p}$, it is also closed and bounded with respect to $d_{p^{\prime}}$. Thus, we can apply the discrete version of Hopf-Rinow Theorem for the graph $\left(\Gamma, d_{p^{\prime}}\right.$ ) (see [Hu-Ke-Ma-Wo], Theorem A1) to conclude that $A$ is finite.

\subsubsection{Trees are regular}

Let $T$ be a locally finite tree with the weight $p$. Let us choose a root $x_{0}$ of $T$.

Definition 4.4 $A$ ray is a maximal simple path in $T$ starting from $x_{0}$.

Let us start with two Lemmas:

Lemma 4.2 The Cauchy boundary of the weighted tree $\left(T, d_{p}\right)$ identifies with the set of rays of finite length with an infinite number of vertices. More precisely, if the ray $\left(x_{0}, x_{1}, \cdots, x_{n}, \cdots\right)$ has a finite length, the sequence $\left(x_{n}\right)$ is a Cauchy sequence. The map $\Phi$ which associates the corresponding point in $T_{\infty}$ to this Cauchy sequence is a bijection.

Proof.-

The map $\Phi$ is injective, because if $\left(x_{n}\right)$ and $\left(y_{l}\right)$ are two rays of finite length the distance $d_{p}\left(x_{n}, y_{l}\right)$ is bounded from below by a strictly positive number as soon as $n$ or $l$ is large enough.

Similarly, if $\left(x_{n}\right)$ is a Cauchy sequence, then $x_{n}$ has to be on a single ray for $n$ large enough. Hence $\Phi$ is surjective.

Lemma 4.3 If all rays of a tree $T$ have a finite number of vertices, $V(T)$ is finite. 
Proof.-

Let us prove the Lemma by contradiction. Let us denote by $|x| \in \mathbb{N}$ the combinatorial distance from $x_{0}$ to $x$. If $V(T)$ is not finite, we can build by induction, using the local finiteness of $T$, a ray $\left(x_{n}\right)_{n \in \mathbb{N}}$ with $\left|x_{n}\right|=n$, so that the number of vertices of the sub-tree rooted at $x_{n}$ and contained in $\{|x| \geq n\}$ is infinite. This ray is infinite, hence a contradiction.

Proposition 4.2 If $T$ is a tree, then for any choice of the weight $p,\left(T, d_{p}\right)$ is regular.

Proof.-

Let us consider a ball $B\left(x_{0}, R\right)$ in $\left(T, d_{p}\right)$. The rays of infinite length are going out of $B\left(x_{0}, R\right)$ after a finite number of steps. The set $X_{\varepsilon}$ is obtained by removing on each infinite ray of finite length all vertices with $|x|$ large enough. Hence, the set $X_{\varepsilon} \cap B\left(x_{0}, R\right)$ is the set of vertices of a tree all of whose rays have a finite number of vertices, and we can apply Lemma 4.3 .

Corollary 4.1 If the first Betti number $b_{1}(G)$ is finite, then $\left(G, d_{p}\right)$ is regular.

Proof.-

Removing a finite number of edges, we get a tree and the sets $X_{\varepsilon}$ are the same for both graphs if $\varepsilon$ is small enough, as well as the bounded sets of $V$.

\subsubsection{An example with a finite Cauchy boundary not satisfying the regularity condition}

Let us consider the following graph $G=(V, E): V=\mathbb{N} \times \mathbb{Z}$,

$$
E=\{\{(n, k),(n+1, k)\} \mid n \in \mathbb{N}, k \in \mathbb{Z}\} \cup\{\{(n, k),(n, k+1)\} \mid n \in \mathbb{N}, k \in \mathbb{Z}\},
$$

and choose the weight $p$ defined as follows:

$$
p_{\{(n, k),(n+1, k)\}}=2^{-(n+1)}, p_{\{(n, k),(n, k+1)\}}=1 / n .
$$


The distance $d_{p}$ satisfies, for $(n, k) \neq\left(n^{\prime}, k^{\prime}\right)$ :

$$
2^{-\left(1+\min \left(n, n^{\prime}\right)\right)} \leq d_{p}\left((n, k),\left(n^{\prime}, k^{\prime}\right)\right) \leq 2^{-n}+2^{-n^{\prime}} \leq 1 .
$$

The lower bound is just the min of the weights of the edges from one of the ends of the path; the upper bound is the limit of the lengths of the curves $\gamma_{N}$ as $N \rightarrow \infty$ where $\gamma_{N}$ is described as follows, assuming that $k^{\prime} \geq k$ and $N>$ $\max \left(n, n^{\prime}\right): \gamma_{N}$ starts from $(n, k)$, follows $(n+1, k),(n+2, k), \cdots,(N, k)$, then $(N, k),(N, k+1), \cdots,\left(N, k^{\prime}\right)$ and finally $\left(N, k^{\prime}\right),\left(N-1, k^{\prime}\right), \cdots,\left(n^{\prime}, k^{\prime}\right)$.

Then $\left(G, d_{p}\right)$ is bounded; a sequence $x_{l}=\left(n_{l}, k_{l}\right)$ is a Cauchy sequence if and only if $n_{l} \rightarrow \infty$; more precisely the diameter of the set $X_{N}:=\{(n, k) \mid n \geq N\}$ is less than $2^{-(N-1)}$. The Cauchy boundary of $\left(G, d_{p}\right)$ is reduced to a single point. The function $D$ is given by

$$
D((n, k))=2^{-n}
$$

Hence the graph $\left(G, d_{p}\right)$ is not regular.

\subsection{Agmon-type estimates}

Lemma 4.4 Let $v, f \in C_{0}(V)$ be real valued and assume $H v=0$. Then

$$
\langle f v, H(f v)\rangle_{l_{\omega}^{2}}=\sum_{\{x, y\} \in E} c_{x, y} v(x) v(y)(f(x)-f(y))^{2} .
$$

Proof.-

In the case of positive $v$ this type of formula is known as ground state transform (see [Hea-Kel] and references within). A particular case of this computation (for operators of the type $\Delta_{1, a}+W$ ) can be found in [To], let us recall the proof for the reader's convenience:

$$
\langle f v, H(f v)\rangle_{l_{\omega}^{2}}=\sum_{x \in V} f(x) v(x)\left(\sum_{y \sim x} c_{x, y}(f(x)-f(y)) v(y)\right)
$$

where we used the fact that $H v(x)=0$. An edge $\{x, y\}$ contributes to the sum twice. The total contribution is

$$
f(x) v(x) c_{x, y}(f(x)-f(y)) v(y)+f(y) v(y) c_{y, x}(f(y)-f(x)) v(x)
$$

so

$$
\langle f v, H(f v)\rangle_{l_{\omega}^{2}}=\sum_{\{x, y\} \in E} c_{x, y}(f(x)-f(y))(f(x) v(x) v(y)-f(y) v(y) v(x)) .
$$


Theorem 4.1 Assume that $\left(G, d_{p}\right)$, with $p_{x y}=\frac{\alpha_{x, y}}{\sqrt{c_{x, y}}}$, is a non complete regular graph. Let $v$ be a solution of $(H-\lambda) v=0$. Assume that $v$ belongs to $l_{\omega}^{2}(V)$ and that there exists a constant $c>0$ such that, for all $u \in C_{0}(V)$,

$$
\langle u \mid(H-\lambda) u\rangle_{l_{\omega}^{2}} \geq \frac{N}{2} \sum_{x \in V} \max \left(\frac{1}{D(x)^{2}}, 1\right) \omega_{x}^{2}|u(x)|^{2}+c\|u\|_{l_{\omega}^{2}}^{2},
$$

then $v \equiv 0$.

Proof.-

This theorem is based on Lemma 4.4 applied to $H-\lambda$. Let us consider $\rho$ satisfying $0<\rho<\frac{1}{2}$. For any $\varepsilon>0$, we define the function $f_{\varepsilon}: V \rightarrow \mathbb{R}$ by $f_{\varepsilon}=F_{\varepsilon}(D)$ where $D$ denotes the distance associated to the metric $d_{p}$, as in (3), and $F_{\varepsilon}: \mathbb{R}^{+} \rightarrow \mathbb{R}$ is the continuous piecewise affine function defined by

$$
F_{\varepsilon}(u)=\left\{\begin{array}{l}
0 \text { for } u \leq \varepsilon \\
\rho(u-\varepsilon) /(\rho-\varepsilon) \text { for } \varepsilon \leq u \leq \rho \\
u \text { for } \rho \leq u \leq 1 \\
1 \text { for } 1 \leq u
\end{array}\right.
$$

Let us fix a vertex $x_{0}$. For any $\alpha>0$, we define also the function $g_{\alpha}: V \rightarrow \mathbb{R}$ by $g_{\alpha}=G_{\alpha}\left(d_{p}\left(x_{0},.\right)\right)$ where $G_{\alpha}: \mathbb{R}^{+} \rightarrow \mathbb{R}$ is the continuous piecewise affine function defined by

$$
G_{\alpha}(u)=\left\{\begin{array}{l}
1 \text { for } u \leq 1 / \alpha \\
-\alpha u+2 \text { for } 1 / \alpha \leq u \leq 2 / \alpha \\
0 \text { for } u \geq 2 / \alpha
\end{array}\right.
$$

Let $E_{\varepsilon, \alpha}$ be the set of the vertices defined by

$$
E_{\varepsilon, \alpha}=\left\{x \in V \mid \varepsilon \leq D(x) \text { and } d_{p}\left(x_{0}, x\right) \leq 2 / \alpha\right\} .
$$

Note that the support of $f_{\varepsilon} g_{\alpha}$ is contained in $E_{\varepsilon, \alpha}$. Additionally, note that $E_{\varepsilon, \alpha}$ is a closed and bounded subset of $X_{\varepsilon}$, where $X_{\varepsilon}$ is as in Definition 4.3. Hence, $E_{\varepsilon, \alpha}$ is finite by Proposition 4.1. Therefore, the function $f_{\varepsilon} g_{\alpha}$ is finitely supported. Observe moreover that

$$
\begin{gathered}
\left|f_{\varepsilon}(x) g_{\alpha}(x)-f_{\varepsilon}(y) g_{\alpha}(y)\right| \leq\left|f_{\varepsilon}(x)\right|\left|g_{\alpha}(x)-g_{\alpha}(y)\right|+\left|g_{\alpha}(y)\right|\left|f_{\varepsilon}(x)-f_{\varepsilon}(y)\right| \\
\leq \frac{\rho}{\rho-\varepsilon}|D(x)-D(y)|+\alpha\left|d_{p}\left(x_{0}, x\right)-d_{p}\left(x_{0}, y\right)\right|
\end{gathered}
$$

so using Lemma 4.1 we get that $f_{\varepsilon} g_{\alpha}$ is $\left(\frac{\rho}{\rho-\varepsilon}+\alpha\right)$-Lipshitz with respect to the metric $d_{p}$. 
We can apply Lemma 4.4 to the finite-supported function $f_{\varepsilon} g_{\alpha}$, and using the inequalities

$$
v(x) v(y) \leq \frac{1}{2}\left(v(x)^{2}+v(y)^{2}\right)
$$

we get that the right hand side of (5) is bounded as follows

$$
\left\langle f_{\varepsilon} g_{\alpha} v \mid(H-\lambda)\left(f_{\varepsilon} g_{\alpha} v\right)\right\rangle_{l_{\omega}^{2}} \leq \frac{1}{2}\left(\frac{\rho}{\rho-\varepsilon}+\alpha\right)^{2} \sum_{x \in V} v(x)^{2} \Phi_{\varepsilon, \alpha}(x),
$$

with

$$
\Phi_{\varepsilon, \alpha}(x)=\sum_{y \sim x} c_{x, y} d_{p}(x, y)^{2} \leq N \omega_{x}^{2}
$$

where the first inequality uses the fact that $f_{\varepsilon} g_{\alpha}$ is $\left(\frac{\rho}{\rho-\varepsilon}+\right.$ $\alpha)$-Lipshitz with respect to the metric $d_{p}$, and the second inequality is a direct consequence of the choice of the weights $p_{x, y}$ (see Lemma 4.1. This implies

$$
\left\langle f_{\varepsilon} g_{\alpha} v \mid(H-\lambda)\left(f_{\varepsilon} g_{\alpha} v\right)\right\rangle_{l_{\omega}^{2}} \leq \frac{N}{2}\left(\frac{\rho}{\rho-\varepsilon}+\alpha\right)^{2}\|v\|_{l_{\omega}^{2}}^{2} .
$$

On the other hand, due to assumption (6) the left hand side of (5) is bounded from below as follows:

$$
\left\langle f_{\varepsilon} g_{\alpha} v \mid(H-\lambda)\left(f_{\varepsilon} g_{\alpha} v\right)\right\rangle_{l_{\omega}^{2}} \geq \frac{N}{2} \sum_{E_{\varepsilon, \alpha}} \omega_{x}^{2} v(x)^{2}+c\left\|f_{\varepsilon} g_{\alpha} v\right\|_{l_{\omega}^{2}}^{2},
$$

where $E_{\varepsilon, \alpha}$ is as in (7).

Putting together (8) and (9) we get

$$
\frac{N}{2} \sum_{E_{\varepsilon, \alpha}} \omega_{x}^{2} v(x)^{2}+c\left\|f_{\varepsilon} g_{\alpha} v\right\|_{l_{\omega}^{2}}^{2} \leq \frac{N}{2}\left(\frac{\rho}{\rho-\varepsilon}+\alpha\right)^{2}\|v\|_{l_{\omega}^{2}}^{2} .
$$

Then we do $\alpha \rightarrow 0$. After that, we do also $\varepsilon \rightarrow 0$. The last step is to take the limit $\rho \rightarrow 0$, and then we get that $v \equiv 0$.

Remark 4.1 The previous result is inspired by a nice idea from [Nen], so following the terminology of [Nen] we call Agmon-type estimates Equation (10). 


\subsection{Essential self-adjointness}

Theorem 4.2 Consider the Schrödinger operator $H=\Delta_{\omega, c}+W$ on a graph $G$, define $\alpha_{x, y}=\min \left\{\omega_{x}, \omega_{y}\right\}$ and assume that $\left(G, d_{p}\right)$, with $p_{x y}=\frac{\alpha_{x, y}}{\sqrt{c_{x, y}}}$, is a non complete regular graph. For a vertex $x \in V$, we denote by $D(x)$ the distance from $x$ to the boundary $V_{\infty}$. We assume the following conditions:

(i) $G$ is of bounded degree and we denote the upper bound by $N$,

(ii) there exists $M<\infty$ so that

$$
\forall x \in V, W(x) \geq \frac{N}{2 D(x)^{2}}-M
$$

Then the Schrödinger operator $H$ is essentially self-adjoint.

Remark 4.2 In the particular case when $\sum \omega_{x}^{2}<\infty$, the Laplacian $H=\Delta_{\omega, c}$ does not satisfy the assumption (11) so this result is coherent with Theorem 3.1.

Remark 4.3 The exponent of $D(x)$ in $\sqrt{11}$ is sharp. In fact, one can find a potential $W$ such that $W(x) \geq \frac{k}{D(x)^{2}}$ where $k<\frac{N}{2}$ and weights $\omega$ and $c$ such that $H=\Delta_{\omega, c}+W$ is non essentially self-adjoint. See Example 5.3.2.

Remark 4.4 In the case where $\omega \equiv 1$ the result is an immediate consequence of [Ke-Le-1] (Theorem 5).

Proof.-

We have, for any $u \in C_{0}(V)$

$$
\langle u \mid H u\rangle_{l_{\omega}^{2}} \geq \sum_{x \in V} W(x) \omega_{x}^{2}|u(x)|^{2},
$$

so using assumption (11) we get:

$$
\langle u \mid(H-\lambda) u\rangle_{l_{\omega}^{2}}-\frac{N}{2} \sum_{x \in V} \frac{1}{D(x)^{2}} \omega_{x}^{2}|u(x)|^{2} \geq \sum_{x \in V}-(M+\lambda)\|u\|_{l_{\omega}^{2}}^{2} .
$$

Then choosing for example

$$
\lambda=-M-1
$$

we get the inequality (6) with $c=1$, and the proof follows from Theorem 4.1. 


\section{Schrödinger operators on "star-like" graphs}

\section{$5.1 \quad$ Introduction}

Definition 5.1 The graph $\mathbb{N}$ is the graph defined by $V=\{0,1,2, \cdots\}$ and $E=$ $\{\{n, n+1\} \mid n=0,1, \cdots\}$.

Definition 5.2 We will call an infinite graph $G=(V, E)$ star-like if there exists a finite sub-graph $G_{0}$ of $G$ so that $G \backslash G_{0}$ is the union of a finite number of disjoint copies $G_{\alpha}$ of the graph $\mathbb{N}$ (the ends of $G$ relatively to $G_{0}$ according to Definition 1.3).

For example, the graph $\mathbb{Z}$, defined similarly to $\mathbb{N}$, is star-like.

Let us consider a Laplace operator $L=\Delta_{1, a}$ on $G$. On each end $G_{\alpha}$ of $G, L$ will be given by

$$
L^{\alpha} f_{n}=-a_{n, n+1}^{\alpha} f_{n+1}+\left(a_{n-1, n}^{\alpha}+a_{n, n+1}^{\alpha}\right) f_{n}-a_{n-1, n}^{\alpha} f_{n-1},
$$

where the $a_{n-1, n}^{\alpha}$ 's are $>0$. If $W: V \rightarrow \mathbb{R}$, we will consider Schrödinger operators $H$ on $C_{0}(G)$ defined by $H=\Delta_{1, a}+W$.

Lemma 5.1 Let $G_{0}$ be a finite sub-graph of $G$. The operator $H=\Delta_{1, a}+W$ on $G$ is essentially self-adjoint if and only if it is essentially self-adjoint on each end of $G$ relatively to $G_{0}$. More precisely, the deficiency indices $n_{ \pm}$are the sum of the corresponding deficiency indices of the ends.

We will need the following Lemma which is a consequence of Kato-Rellich Theorem, see [Go-Sch], Proposition 2.1:

Lemma 5.2 If $A$ and $B$ are 2 symmetric operators with the same domains and $R=B-A$ is bounded, then the deficiency indices of $A$ and $B$ are the same.

Proof.-

We give here an alternative proof to this result. Let us define, for $t \in \mathbb{R}, A_{t}=A+t R$ so that $A_{0}=A$ and $A_{1}=B$. The domains of the closures of the $A_{t}$ 's coincide: the "graph-norms" $\left\|A_{t} u\right\|_{l^{2}}+\|u\|_{l^{2}}$ are equivalent. The domains of the adjoints coincide too. Let $K=$ $D\left(A^{\star}\right) / D(\bar{A})$ and $Q_{t}(u, v)=-i\left(\left\langle A_{t}^{\star} u \mid v\right\rangle-\langle u|\left(A_{t}^{\star} v\right\rangle\right)$ which is well defined on $K$. We know that these bounded Hermitian forms are non degenerate on $K$ with the graph norm and continuous w.r. to $t$. Hence the Morse index $n_{-}(t)$ is locally constant: take a decomposition $K=$ $K_{+} \oplus K_{-}$where $q=Q_{t_{0}}$ satisfies $q_{\mid K_{+}} \geq C>0$ and $q_{\mid K_{-}} \leq-C<0$.

Using Lemma 5.2, we can prove Lemma 5.1; Proof.- 
We will consider the operator $H_{\text {red }}$ where we replace the entries $a_{x, y}$ of $H$ with $\{x, y\} \in E\left(G_{0}\right)$ by 0 . The claim of the Lemma is clear for $H_{\text {red }}$ because it is the direct orthogonal sum of the Schrödinger operators of the ends and a finite rank $l^{2}$-bounded matrix. We can then use Lemma 5.2 because $H-H_{\text {red }}$ is bounded.

Remark 5.1 It follows from Lemma 5.1 that, concerning essential self-adjointness questions for star-like graphs, it is enough to work on the graph $\mathbb{N}$. We have

$$
(H f)_{0}=-a_{0,1} f_{1}+a_{0,1} f_{0}+W_{0} f_{0} .
$$

This implies that the space of solutions of $(H-\lambda) u=0$ on $\mathbb{N}$ is of dimension 1 and any solution so that $f_{0}$ vanishes is $\equiv 0$. We will consider also solutions "near infinity", i.e. $\left(f_{n}\right)_{n \geq 0}$ satisfies $((H-\lambda) f)_{n}=0$ for $n \geq 1$; this space is of dimension 2.

\subsection{Main result}

It is known $([\overline{\mathrm{Dod}}])$ that $H=\Delta_{1, a}+W$ is essentially self-adjoint provided $\Delta_{1, a}$ is bounded as an operator on $l^{2}(G)$ and $W$ bounded from below. For star-like graphs, we have the following result, which holds for any potential $W$ :

Theorem 5.1 If $G$ is star-like and if for each end $G_{\alpha}$,

$$
1 / a_{n-1, n}^{\alpha} \notin l^{1}(\mathbb{N})
$$

then $H=\Delta_{1, a}+W$ with domain $C_{0}(V)$ is essentially self-adjoint for any potential $W$.

Remark 5.2 The condition (12) is sufficient but not necessary. See Example 5.3 .2 .

Proof.-

Due to Remark 5.1 we only have to prove the following

Theorem 5.2 If

$$
\frac{1}{a_{n-1, n}} \notin l^{1}(\mathbb{N}),
$$

the Schrödinger operator $H=\Delta_{1, a}+W$ with domain $C_{0}(\mathbb{N})$ is essentially self-adjoint for any potential $W$. 
This result is contained in the book $[\mathrm{Ber}$ (p. 504). We propose here a short proof, obtained by contradiction using Corollary 6.1 which is an analog of Weyl's limit point-limit circle criteria in the discrete case.

Let us consider an operator $\Delta_{1, a}$ such that $(12)$ is fulfilled. We assume that any sequence $u$, such that $(H-i) u=0$ near infinity, is in $l^{2}(\mathbb{N})$. In particular, there exists a basis $f, g$ of solutions of $(H-i) f=0$ with $f \in l^{2}(\mathbb{N})$ and $g \in l^{2}(\mathbb{N})$.

We have

$$
-a_{n, n+1} f_{n+1}+\left(a_{n-1, n}+a_{n, n+1}+\left(W_{n}-i\right)\right) f_{n}-a_{n-1, n} f_{n-1}=0,
$$

and the same holds for $g$. The Wronskian of $f$ and $g$ is the sequence $\mathcal{W}_{n}=f_{n} g_{n-1}-f_{n-1} g_{n}$. We have, for any $n \in \mathbb{N}$ :

$$
\mathcal{W}_{n+1}=\frac{a_{n-1, n}}{a_{n, n+1}} \mathcal{W}_{n}
$$

which implies

$$
\mathcal{W}_{n}=\frac{a_{0,1}}{a_{n-1, n}} \mathcal{W}_{1}
$$

But since the Wronskian is in $l^{1}(\mathbb{N})$ according to the assumption that $f$ and $g$ are in $l^{2}(\mathbb{N})$, we get a contradiction with the hypothesis 12 .

\subsection{Examples of Schrödinger operators}

\subsubsection{Example 1}

Let us consider the Laplacian $\Delta_{\omega, c}$ on $\mathbb{N}$, with, $\forall n>0, c_{n-1, n}=n^{3}$ and, $\forall n \geq 0$, $\omega_{n}=\frac{1}{n+1}$. Since $\sum \omega_{n}^{2}<\infty$ and $\sum c_{n-1, n}^{-1 / 2}<\infty$ we deduce from Theorem 3.1 (due to Rem 3.1) that $\Delta_{\omega, c}$ is not essentially self-adjoint.

Applying a result of [To] (see Proposition 7.1] in Appendix B) we get that this Laplacian is unitarily equivalent to the Schrödinger operator $H=\Delta_{1, a}+W$ with $a_{n-1, n}=\frac{c_{n-1, n}}{\omega_{n-1} \omega_{n}} \sim n^{5}$ and

$$
W_{n}=\frac{1}{\omega_{n}}\left[c_{n, n+1}\left(\frac{1}{\omega_{n}}-\frac{1}{\omega_{n+1}}\right)+c_{n-1, n}\left(\frac{1}{\omega_{n}}-\frac{1}{\omega_{n-1}}\right)\right] \sim-3 n^{3},
$$

which is therefore not essentially self-adjoint.

According to Theorem 5.1. such an operator must verify $\frac{1}{a_{n-1, n}} \in l^{1}(\mathbb{N})$, which is indeed the case. 


\subsubsection{Example 2: Discretization of a Schrödinger operator on $\mathbb{R}^{+}$}

Let us consider the Schrödinger operator on $] 0,+\infty[$ defined on smooth compactly supported functions by $L f:=-f "+\frac{A}{x^{2}} f$. This operator is essentially self-adjoint if and only if $A>3 / 4$ (see [RS] theorem X 10). We discretize this operator in the following way: let us consider the graph $\Gamma=(V, E)$ resulting of the following dyadic subdivision of the interval $(0,1)$ : the vertices are the $x_{n}=2^{-n}$ and the edges are the pairs $\left\{2^{-n}, 2^{-n+1}\right\}$ which correspond to the intervals $\left[2^{-n}, 2^{-n+1}\right]$ of length $\omega_{n}^{2}=2^{-n}$.

Then we define, for any

$$
f \in l_{\omega}^{2}(V)=\left\{f \in C(V) \mid \sum_{n \in \mathbb{N}} 2^{-n} f_{n}^{2}<+\infty\right\}
$$

where we set $f=\left(f_{n}\right)$, the quadratic form

$$
Q(f)=\sum_{n \in \mathbb{N}} 2^{-n}\left[\left(\frac{f_{n+1}-f_{n}}{2^{-n}}\right)^{2}+A 2^{2 n} f_{n}^{2}\right] .
$$

According to the previous definitions and if we set $c_{n, n+1}=2^{n}$, this quadratic form is associated to the Schrödinger operator $H=\Delta_{\omega, c}+W$ on $\mathbb{N}$ with the potential $W_{n}:=A 2^{2 n}$.

Let us set $a_{n, n+1}=\frac{c_{n, n+1}}{\omega_{n} \omega_{n+1}}=2^{2 n+\frac{1}{2}}$. Applying Proposition 7.1 we get that $H$ is unitarily equivalent to

$$
\widehat{H}=\Delta_{1, a}+\widehat{W}+W
$$

with

$$
\widehat{W}_{n}=\frac{1}{\omega_{n}}\left[c_{n, n+1}\left(\frac{1}{\omega_{n}}-\frac{1}{\omega_{n+1}}\right)+c_{n-1, n}\left(\frac{1}{\omega_{n}}-\frac{1}{\omega_{n-1}}\right)\right]=2^{2 n}\left(\frac{3}{2}-\frac{5 \sqrt{2}}{4}\right) .
$$

We have $\widehat{H}=\Delta_{1, a}+\left(A-A_{0}\right) 4^{n}$ with $A_{0}=\frac{5 \sqrt{2}}{4}-\frac{3}{2}(>0)$. The metric graph $\left(\mathbb{N}, d_{p}\right)$ with $p_{n, n+1}=a_{n, n+1}^{-1 / 2}$ is non complete. The solutions $u$ of $H u=0$ verify

$$
4 u_{n+1}-\left(5+2 \sqrt{2}\left(A-A_{0}\right)\right) u_{n}+u_{n-1}=0 .
$$

The solutions are generated by $\alpha_{1}^{n}$ and $\alpha_{2}^{n}$ where $\alpha_{1}$ and $\alpha_{2}$ are the roots of

$$
4 \alpha^{2}-\left(5+2 \sqrt{2}\left(A-A_{0}\right)\right) \alpha+1=0 .
$$

We have $\left|\alpha_{1}\right|<1$ and $\left|\alpha_{2}\right|<1$ if and only if $A_{0}-\frac{5}{\sqrt{2}}<A<A_{0}$. 
Using Proposition 6.1, with $d=2$ and $U_{n}=\left(\begin{array}{c}u_{n} \\ u_{n-1}\end{array}\right)$, we get, for any $\lambda \in \mathbb{C}$, the exponential decay of all solutions near infinity of $(\widehat{H}-\lambda) u=0$ if $A_{0}-\frac{5}{\sqrt{2}}<A<A_{0}$, and the existence of a solution of $(\widehat{H}-\lambda) u=0$ with exponential growth in the case when $A>\frac{5 \sqrt{2}}{4}-\frac{3}{2}$ or $A<-\frac{5 \sqrt{2}}{4}-\frac{3}{2}$.

Hence (by Corollary 6.1) we get the following result:

Proposition 5.1 1. If $-\frac{5 \sqrt{2}}{4}-\frac{3}{2}<A<\frac{5 \sqrt{2}}{4}-\frac{3}{2}$, then the discretized operator $H$ is not essentially self-adjoint.

2. If $A>\frac{5 \sqrt{2}}{4}-\frac{3}{2}(\star) \quad$ or $A<-\frac{5 \sqrt{2}}{4}-\frac{3}{2}$, then $H$ is essentially self-adjoint.

From this result we can deduce several informations:

1. The condition $(\star)$ is analogous to the condition $A>3 / 4$ in the continuous case.

2. Proposition 5.1 implies that for $A=0$ the operator $H=\Delta_{\omega, c}$ is not essentially self-adjoint, which is a result predicted by Theorem 3.1 .

3. This gives examples of essentially self-adjoint operators with $1 / a_{n} \in l^{1}$.

4. Sharpness of the assumption (11) in Theorem 4.2

In this context, the distance $d_{p}$ is associated to

$$
p_{x, y}=\frac{\alpha_{x, y}}{\sqrt{c_{x, y}}}
$$

with $\alpha_{x, y}=\min \left\{\omega_{x}, \omega_{y}\right\}$ so we get

$$
D(n)=\sum_{p \geq n} \frac{\alpha_{p, p+1}}{\sqrt{c_{p, p+1}}}=\sum_{p \geq n}\left(\frac{2^{-p-1}}{2^{p}}\right)^{1 / 2}=2^{-\frac{1}{2}} 2^{-n} 2
$$

So

$$
\frac{1}{D(n)^{2}}=2^{2 n-1}
$$

If the operator $H=\Delta_{\omega, c}+A 4^{n}$ satisfies the assumption (11), then $A>\frac{1}{2}$ which involves condition $(\star)$, since $\frac{1}{2}>\frac{5 \sqrt{2}}{4}-\frac{3}{2}$, so Theorem 4.2 is coherent with proposition 5.1. Moreover the operator $H=\Delta_{\omega, c}+A 4^{n}$ with $A=\frac{5 \sqrt{2}}{4}-\frac{3}{2}$ is not essentially self-adjoint, which implies that the estimate (11) on the growth of the potential in Theorem 4.2 is sharp. 


\subsubsection{Example 3}

Let us consider the Laplacian $\Delta_{\omega, c}$ on $\mathbb{N}$, where the coefficients verify $c_{n-1, n}=n^{\gamma}$ with $\gamma>2$ and $\omega_{n}=(n+1)^{-\beta}$ with $\beta>\frac{1}{2}$. Since $\sum \omega_{n}^{2}<\infty$ and $\sum c_{n-1, n}^{-1 / 2}<\infty$ we deduce from Theorem 3.1 (due to Remark 3.1 ) that $\Delta_{\omega, c}$ is not essentially self-adjoint.

Applying one more time Proposition 7.1, we see that this operator is unitarily equivalent to the Schrödinger operator $H=\Delta_{1, a}+W$, with $a_{n-1, n} \sim n^{\gamma+2 \beta}$ and the potential $W_{n} \sim-\beta(\beta+\gamma-1) n^{2 \beta+\gamma-2}$, which is therefore also not essentially self-adjoint. We emphasize that $W$ is not bounded from below, which is predicted in [To], Theorem 3.2.

Furthermore, according to Theorem 5.1, such an operator must verify the condition $\frac{1}{a_{n-1, n}} \in l^{1}(\mathbb{N})$, which is indeed the case. Following the terminology of the previous sections, it means the non completeness of $\left(\mathbb{N}, d_{p}\right)$ with the weights $p_{n-1, n}=a_{n-1, n}^{-1 / 2}$.

\subsubsection{Example 4}

Let us consider the Laplacian $H=\Delta_{\omega, c}$ on a spherically homogeneous rooted tree $G=(V, E)$ (see [Bre] and references within). For any vertex $x$, we denote by $\delta(x)$ the distance from $x$ to the root 0 and define $\omega_{x}=2^{-\delta(x)}$, and $c_{x, y}=2^{\delta(x)}$, for any $y \sim x$ so that $\delta(y)=n+1$. We assume that the graph $G$ has a uniform degree $N+1$.

Let us set $a_{x, y}=\frac{c_{x, y}}{\omega_{x} \omega_{y}}$. We have $a_{x, y}=2^{3 n+1}$ for any edge $x, y$, so that $\delta(x)=n$ and $\delta(y)=n+1$. Then, due to Proposition 7.1, the operator $H$ is unitarily equivalent to

$$
\widehat{H}=\Delta_{1, a}+W
$$

with

$$
W(x)=2^{3 n}\left(-N+\frac{1}{4}\right)
$$

for any $x$ such that $\delta(x)=n$.

The radial solutions $u$ of $H u=0$ can be seen as sequences $\left(u_{n}\right)$ which satisfy the equation:

$$
-2 N u_{n+1}+\left(N+\frac{1}{2}\right) u_{n}-\frac{1}{4} u_{n-1}=0 .
$$

The solutions are generated by $\alpha_{1}^{n}$ and $\alpha_{2}^{n}$ where $\alpha_{1}$ and $\alpha_{2}$ are the roots of

$$
\alpha^{2}-\left(\frac{1}{2}-\frac{1}{4 N}\right) \alpha+\frac{1}{8 N}=0 \text {. }
$$

We have $\left|\alpha_{1}\right|<1$ and $\left|\alpha_{2}\right|<1$ for any $N>0$. 
The radial solutions of $(\widehat{H}-\lambda) u=0$ satisfy

$$
-2 N u_{n+1}+\left(N+\frac{1}{2}\right) u_{n}-\frac{1}{4} u_{n-1}=2 N \lambda 2^{-(3 n+1)} u_{n} .
$$

Using Proposition 6.1, with $d=2$ and $U_{n}=\left(\begin{array}{c}u_{n} \\ u_{n-1}\end{array}\right)$, we get the exponential decay of all solutions near infinity of $(\widehat{H}-\lambda) u=0$.

Hence (by Corollary 6.1) we get the following result:

Proposition 5.2 For any $N \geq 1 H$ is not essentially self-adjoint.

Remark 5.3 We have

$$
\sum_{x} \omega_{x}^{2}=\sum_{n} \omega_{n}^{2} N^{n}=\sum_{n}\left(\frac{N}{4}\right)^{n} .
$$

If $N<4$, then $\sum_{x} \omega_{x}^{2}<\infty$ so Theorem 3.1 can also be applied to get the result since the graph is non complete with respect to the metric $d_{p}$, with $p_{x, y}=c_{x, y}^{-\frac{1}{2}}$.

\section{Appendix A: Weyl's "limit point-limit circle" criteria}

\subsection{The discrete case}

The goal of this section is to prove the discrete version of the Weyl's "limit pointlimit circle" criterium. Our presentation is simpler than the classical presentation for the continuous case (see [RS], Appendix to section X.1).

Let us consider the Hilbert space $\mathcal{H}:=l^{2}\left(\mathbb{N}, \mathbb{C}^{N}\right)$ and the formally symmetric differential operator $P$ defined by

$P f(0)=P_{0,0} f(0)+P_{0,1} f(1), \forall l \geq 1, P f(l)=P_{l, l-1} f(l-1)+P_{l, l} f(l)+P_{l, l+1} f(l+1)$

where

1. $\forall l \geq 1, P_{l-1, l}^{\star}=P_{l, l-1}$

2. $\forall l \geq 0, P_{l, l}^{\star}=P_{l, l}$

3. $\forall l \geq 0, P_{l, l+1}$ is invertible

4. $\exists M \in \mathbb{R}$ so that for any $f \in C_{0}\left(\mathbb{N}, \mathbb{C}^{N}\right), Q_{P}(f)=\langle P f \mid f\rangle \geq-M\|f\|^{2}$. 
Let us define the subspace $\mathcal{E}$ of $\mathcal{H}$ as the set of $l^{2}$ sequences $f$ so that, for all $l \geq 1,(P-i) f(l)=0$; the space $\mathcal{E}$ is isomorphic to the space of germs at infinity of $l^{2}$ solutions of $(P-i) f=0$. Assumption 3. implies that $\operatorname{dim} \mathcal{E} \leq 2 N$. Let us denote by $\mathcal{K}=\operatorname{ker}(P-i) \cap l^{2}$ and consider the following sequence

$$
0 \rightarrow \mathcal{K} \rightarrow \mathcal{E} \rightarrow \mathbb{C}^{N} \rightarrow 0
$$

where the non trivial arrow is given by $f \rightarrow(P-i) f(0)$. We have the

Theorem 6.1 The sequence (14) is exact and the deficiency indices $n_{ \pm}=\operatorname{dim} \mathcal{K}$ of $P$ are given by $n_{ \pm}=\operatorname{dim} \mathcal{E}-N$.

Proof.-

Assumption 4. implies (using Corollary of Theorem X.1 in [RS]) that the deficiency indices are equal. The only non trivial point is to prove that the arrow $p: \mathcal{E} \rightarrow \mathbb{C}^{N}$ is surjective. Let us consider $\tilde{P}$ a selfadjoint extension of $P$ which exists because $n_{+}=n_{-}$. Let us consider the map $\rho: \mathbb{C}^{N} \rightarrow \mathcal{E}$ defined by $\rho(x)=(\tilde{P}-i)^{-1}(x, 0,0, \cdots)$. Then $p \circ \rho=\operatorname{Id}_{\mathbb{C}^{N}}$.

Corollary 6.1 The Schrödinger operator $H=\Delta_{1, a}+W$ defined on $C_{0}(\mathbb{N})$ is essentially self-adjoint if and only if there exists a sequence $u$ such that $(H-i) u=$ 0 near infinity (i.e. $((H-i) u)_{n}=0$ for $n$ large enough) which is not in $l^{2}(\mathbb{N})$.

\subsection{Asymptotic behavior of perturbed hyperbolic itera- tions}

In order to apply Corollary 6.1, the following results will be useful

Proposition 6.1 Let us consider the following linear dynamical system on $\mathbb{C}^{d}$ :

$$
\forall n \geq 0, U_{n+1}=A U_{n}+R(n) U_{n}
$$

where

1. A is hyperbolic: all eigenvalues $\lambda_{j}$ of $A$ satisfy $\left|\lambda_{j}\right| \neq 1$

2. $R(n) \rightarrow 0$ as $n \rightarrow \infty$.

Then

- case A: If all eigenvalues $\lambda_{j}$ of $A$ satisfy $\left|\lambda_{j}\right|<1$, all solutions $\left(U_{n}\right)$ of Equation 15) are exponentially decaying. 
- case B: If m eigenvalues satisfy $\left|\lambda_{j}\right|>1$, then there exists an m-dimensional vector space $F$ of solutions of Equation (15) whose non-zero vectors have exponential growth.

Proof.-

Case A: There exists a norm $\|\cdot\|$ on $\mathbb{C}^{d}$ so that the operator norm of $A$ satisfies $\|A\|=k<1$. For $n$ large enough, we have $\|A+R(n)\| \leq k^{\prime}<1$. The conclusion follows.

Case B: There exists a splitting $\mathbb{C}^{d}=Y \oplus Z$, denoted $x=y+z$, with $\operatorname{dim} Y=m$, stable by $A$, norms on $Y$ and $Z$ and 2 constants $\mu<1<\sigma$, so that

$$
\begin{aligned}
& \forall y \in Y,\|A y\| \geq \sigma\|y\|, \\
& \forall z \in Z,\|A z\| \leq \mu\|z\| .
\end{aligned}
$$

Let us choose $\varepsilon>0$ so that $1<\sigma-2 \varepsilon$ and $N$ so that $\|R(n)\| \leq \varepsilon$ for $n \geq N$. We have, for $n \geq N$,

$$
\left\|y_{n+1}\right\| \geq \sigma\left\|y_{n}\right\|-\varepsilon\left(\left\|y_{n}\right\|+\left\|z_{n}\right\|\right),\left\|z_{n+1}\right\| \leq \mu\left\|z_{n}\right\|+\varepsilon\left(\left\|y_{n}\right\|+\left\|z_{n}\right\|\right),
$$

so that

$$
\left\|y_{n+1}\right\|-\left\|z_{n+1}\right\| \geq(\sigma-2 \varepsilon)\left(\left\|y_{n}\right\|-\left\|z_{n}\right\|\right) .
$$

Any solution which satisfies $\left\|y_{N}\right\|>\left\|z_{N}\right\|$ will have exponential growth.

Take for $F$ the space of solutions for which $z_{N}=0$.

\subsection{The continuous case}

A similar method works for the continuous case. Let $H=-\frac{d^{2}}{d x^{2}}+A(x)$ be a system of differential operators where $A(x)$ is Hermitian for every $x$ and is continuous on $\left[0, a\left[\right.\right.$ as a function of $x$. The differential operator $H$ is $L^{2}$-symmetric on the Dirichlet domain

$$
D=C_{0}^{\infty}\left(\left[0, a\left[, \mathbb{C}^{N}\right) \cap\{u \mid u(0)=0\} .\right.\right.
$$

We denote $H_{D}$ the closure of $(H, D)$. Let us assume that $n_{+}\left(H_{D}\right)=n_{-}\left(H_{D}\right)$ which is true for example if $A$ is bounded from below or if $A$ is real-valued. Then

Theorem 6.2 If $\mathcal{E}$ is the space of solutions $u$ of the differential equation $(H-i) u=0$ which are $L^{2}$ near a, then $n_{ \pm}\left(H_{D}\right)=\operatorname{dim} \mathcal{E}-N$.

Proof.- 
Let us consider the sequence

$$
0 \rightarrow \operatorname{ker}\left(H_{D}-i\right) \rightarrow \mathcal{E} \rightarrow \mathbb{C}^{N} \rightarrow 0,
$$

where the only non trivial arrow is given by $u \rightarrow u(0)$. This sequence is exact: we have only to prove the surjectivity of the non trivial arrow. Let $\tilde{H}$ be a self-adjoint extension of $H_{D}$ and $\chi \in C_{0}^{\infty}([0, a[, \mathbb{R})$ with $\chi(0)=1$. For any $X \in \mathbb{C}^{N}$, let us consider

$$
u=\chi X-(\tilde{H}-i)^{-1}((H-i)(\chi X)) .
$$

Then $(H-i) u=0, u(0)=V$ and $u$ is $L^{2}$ near $a$.

\section{Appendix B: Unitary equivalence between Lapla- cians and Schrödinger operators}

In this section, we recall the following results (see [To] Proposition 2.1 and Theorem 5.1): the first one states that a Laplacian is always unitarily equivalent to a Schrödinger operator, and the second result asserts that a Schrödinger operator with a strictly positive quadratic form is unitarily equivalent to a Laplacian.

For a weighted graph $G$ by the weight $\omega$ on its vertices, let

$$
U_{\omega}: l_{\omega}^{2}(V) \longrightarrow l^{2}(V)
$$

the unitary operator defined by

$$
U_{\omega}(f)=\omega f .
$$

This operator preserves the set of functions on $V$ with finite support.

Proposition 7.1 The operator

$$
\widehat{\Delta}=U_{\omega} \Delta_{\omega, c} U_{\omega}^{-1},
$$

is a Schrödinger operator on $G$. More precisely:

$$
\widehat{\Delta}=\Delta_{1, a}+W
$$

where $a$ is a strictly positive weight on $E$ given by:

$$
a_{x, y}=\frac{c_{x, y}}{\omega_{x} \omega_{y}}
$$

and the potential $W: V \longrightarrow \mathbb{R}$ is given by:

$$
W=-\frac{1}{\omega} \Delta_{1, a} \omega .
$$


The following Theorem uses the existence of a strictly positive harmonic function (see [To], section 4).

Theorem 7.1 Let $P$ a Schrödinger operator on a graph $G$. We assume that $\langle P f, f\rangle_{l^{2}}>0$ for any function $f$ in $C_{0}(V) \backslash\{0\}$. Then there exist weights: $\omega$ on $V$ and $c$ on $E$ such that $P$ is unitarily equivalent to the Laplacian $\Delta_{\omega, c}$ on the graph $G$.

\section{Acknowledgments}

We would like to thank Ognjen Milatovic, who pointed out the existence of some gap in the proof of Theorem 4.1 of our early version of the paper. In order to remove this gap, we have made an additional assumption on the graph, which is a regularity property, and which allows us to prove the finiteness of the support of the cut off functions in Theorem 4.1.

The second author is greatly indebted to the research unity "Mathématiques et Applications" (05/UR/15-02) of Faculté des Sciences de Bizerte (Tunisie) for the financial support, and would like to present special thanks to Institut Fourier where this work was carried on.

Thanks to D. Lenz for giving notes on some references.

All the authors would like to thank the reviewers for their comments and especially Reviewer 1 for careful reading, numerous remarks, useful suggestions and valuable references.

\section{References}

[Ber] Ju.M. Berezans'kii. Expansions in eigenfunctions of selfadjoint operators. Translations of Mathematical Monographs 17, American Mathematical Society, Providence, R.I. (1968).

[B-M-S] M. Braverman, O. Milatovic \& M. Shubin. Essential self-adjointness of Schrödinger-type operators on manifolds, Russian Math. Surveys 57 (2002) 641-692 .

[Bre] J. Breuer. Singular continuous spectrum for the Laplacian on certain sparse trees, Commun. Math. Phys. 269 (3) (2007) 851-857.

[Col1] Y. Colin de Verdière. Pseudos-Laplaciens I, Ann. Inst. Fourier (Grenoble), 32 (1982) 275-286.

[Col2] Y. Colin de Verdière. Spectre de graphes, Cours spécialisés 4, Société mathématique de France (1998). 
[Col-Tr] Y. Colin de Verdière \& F. Truc. Confining quantum particles with a purely magnetic field, Ann. Inst. Fourier (Grenoble) (to appear).

[Dod] J. Dodziuk. Elliptic operators on infinite graphs, Analysis geometry and topology of elliptic operators, 353-368, World Sc. Publ., Hackensack NJ. (2006).

[Du-Sc] N. Dunford \& J. T. Schwartz. Linear operator II, Spectral Theory, John Wiley \& Sons, New York (1971).

[Go-Sch] S. Golénia \& C. Schumacher. The problem of deficiency indices for discrete Schrödinger operators on locally finite graphs, arXiv:1005.0165 (2010).

[Hea-Kel] S. Haeseler \& M. Keller. Generalised solutions and spectrum for Dirichlet forms on graphs, arXiv:1002.1040 (2010).

[Hu] X. Huang. A note on stochastic incompletness for graphs and weak OmoriYau maximum principle, arXiv:1009.2579 (2010).

[Hu-Ke-Ma-Wo] X. Huang, M. Keller, J. Masamune \& R.K. Wojciechowski. A note on self-adjoint extensions of the Laplacian on weighted graphs, arXiv:1208.6538

[Jor] P.E.T. Jorgensen. Essential self-adjointness of the graph-Laplacian, J. Math. Phys. 49 (7) (2008) 073510, 33pp.

[Jo-Pe-1] P.E.T. Jorgensen \& E.P.J. Pearse. Spectral reciprocity and matrix representations of unbounded operators, arXiv:0911.0185 (2009)

[Jo-Pe-2] P.E.T. Jorgensen \& E.P.J. Pearse. A discrete Gauss-Green identity for unbounded Laplace operators, and the transience of Random walks, arXiv:0906.1586 (2010).

[Ke-Le-1] M. Keller \& D. Lenz. Dirichlet forms and stochastic completneness of graphs and subgraphs, arXiv:0904.2985 (2009).

[Ke-Le-2] M. Keller \& D. Lenz. Unbounded Laplacians on graphs: basic spectral properties and the heat equation, Math. Nat. Phenomena, 5 (2010), n 4.

[Nen] G. Nenciu \& I. Nenciu. On confining potentials and essential selfadjointness for Schrödinger operators on bounded domains in $\mathbb{R}^{n}$, Ann. Henri Poincaré, 10 (2009), 377-394.

[Ma] J. Masamune. A Liouville property and its application to the Laplacian of an infinite graph, Contemp. Math., 484 (2009), 103-115, 
[Ol] I.M. Oleinik. On the essential self-adjointness of the operator on complete Riemannian manifolds, Mathematical Notes 54 (1993), 934-939 .

[RS] M.Reed \& B.Simon. Methods of Modern mathematical Physics, I-Functional analysis,(1980), II-Fourier analysis, Self-adjointness, (1975). New York Academic Press.

[Shu1] M. Shubin. The essential self-adjointness for semi-bounded magnetic Schrödinger operators on non-compact manifolds, J. Func. Anal. 186 (2001), 92-116.

[Shu2] M. Shubin. Classical and quantum completness for the Schrödinger operators on non-compact manifolds, Geometric Aspects of Partial Differential Equations (Proc. Sympos., Roskilde, Denmark (1998)) Amer. Math. Soc. Providence, RI, 257-269 (1999).

[To] N. Torki-Hamza. Laplaciens de graphes infinis I Graphes métriquement complets, Confluentes Mathematici 2 (2010), n 3.

[We] A. Weber. Analysis of the physical Laplacian and the heat flow on a locally finite graph, J. Math. Anal. Appl. 370 (2010), 146-158.

[Woj1] R.K. Wojiechowski. Stochastic completeness of graphs, Ph.D. Thesis, The graduate Center of the University of New-York (2008).

[Woj2] R.K. Wojiechowski. Heat kernel and essential spectrum of infinite graphs, Univ. Math. J. 58 (2009), n 3, 1419-1442. 